\title{
Baseline Characteristics and Treatment Patterns of the Patients Recruited to the China Registry of Hepatitis B
}

\author{
Shan Shan ${ }^{1}$, Hong You ${ }^{1}$, Junqi Niu ${ }^{2}$, Jia Shang ${ }^{3}$, Wen Xie ${ }^{4}$, Yuexin Zhang ${ }^{5}$, Xun $\mathrm{Li}^{6}$, Hong Ren ${ }^{7}$, \\ Hong Tang ${ }^{8}$, Huiguo Ding ${ }^{9}$, Xihong Wang ${ }^{10}$, Yuemin $\mathrm{Nan}^{11}$, Xiaoguang Dou ${ }^{12}$, Tao Han ${ }^{13}$ \\ Lingyi Zhang ${ }^{14}$, Xiaoqing Liu ${ }^{15}$, Cunliang Deng ${ }^{16}{ }^{\prime}$ Jilin Cheng $^{17}$, Xiaozhong Wang ${ }^{18}$, Qing Xie ${ }^{19}$, \\ Shumei Lin ${ }^{20}$, Yan Huang ${ }^{21}$, Youqing Xu' ${ }^{22}$, Yong Xiong ${ }^{23}, \mathrm{Wu} \mathrm{Li}^{24}$, Xuebing Yan ${ }^{25}$, \\ Hongxin Piao ${ }^{26}$, Wenxiang Huang ${ }^{27}$, Qinghua Lu ${ }^{28}$, Weijin Gong ${ }^{29}$, Shiping $\mathrm{Li}^{30}$, \\ Xiaoxuan $\mathrm{Hu}^{31}$, Xiaolan Zhang ${ }^{32}$, Shourong Liu ${ }^{33}$, Yufang $\mathrm{Li}^{34}$, Dongliang Yang ${ }^{35}$, $\mathrm{Hai} \mathrm{Li}^{36}$, \\ Caixia Yang ${ }^{37}$, Mingliang Cheng ${ }^{38}$, Liaoyun Zhang ${ }^{39}$, Huanwei Zheng ${ }^{40}$, Xinhua Luo ${ }^{41}$, \\ Feng Lin ${ }^{42}$, Lei Wang ${ }^{43}$, Guanghua $\mathrm{Xu}^{44}$, Xiaoyuan $\mathrm{Xu}^{45}$, Lai Wei ${ }^{46}$, Jinlin Hou ${ }^{47}$, \\ Zhongping Duan ${ }^{48}$, Hui Zhuang ${ }^{49}$, Xizhong Yang ${ }^{50}$, Yuanyuan 'Kong ${ }^{51 *}$, and Jidong Jia ${ }^{1 *}$ for \\ the CR-HepB study group, Beijing, China
}

${ }^{1}$ Liver Research Center, Beijing Friendship Hospital, Capital Medical University, Beijing Key Laboratory of Translational Medicine on Liver Cirrhosis, National Clinical Research Center for Digestive Diseases, Beijing, China; ${ }^{2}$ Department of Hepatology, First Hospital of Jilin University, Changchun, Jilin, China; ${ }^{3}$ Department of Infectious Diseases, Henan Provincial People's Hospital, Zhengzhou, Henan, China; ${ }^{4}$ Center of Liver Diseases, Beijing Ditan Hospital, Capital Medical University, Beijing, China;

${ }^{5}$ Department of Infectious Diseases, First Affiliated Hospital of Xinjiang Medical University, Urumqi, Xinjiang, China; ${ }^{6}$ Department of General Surgery, The First Hospital of Lanzhou University, Lanzhou, Gansu, China; ${ }^{7}$ Department of Infectious Diseases, The Second Affiliated Hospital of Chongqing Medical University, Chongqing, China; ${ }^{8}$ Center of Infectious Diseases, West China Hospital of Sichuan University, Chengdu, Sichuan, China; ${ }^{9}$ Department of Gastroenterology and Hepatology, Beijing Youan Hospital, Capital Medical University, Beijing, China; ${ }^{10}$ Center of Infectious Diseases, The Third People's Hospital of Taiyuan,

Taiyuan, Shanxi, China; ${ }^{11}$ Department of Traditional and Western Medical Hepatology, The Third Hospital of Hebei Medical University, Shijiazhuang, Hebei, China; ${ }^{12}$ Department of Infectious Diseases, Shengjing Hospital of China Medical University, Shenyang, Liaoning, China; ${ }^{13}$ Department of Hepatology, Tianjin Third Central Hospital, Tianjin Medical University, Tianjin, China; ${ }^{14}$ Department of Hepatology, Lanzhou University Second Hospital, Lanzhou, Gansu, China; ${ }^{15}$ Department of Infectious Diseases, Peking Union Medical College Hospital, Beijing, China; ${ }^{16}$ Department of Infectious Diseases, The Affiliated Hospital of Southwest Medical University, Luzhou, Gansu, China; ${ }^{17}$ Department of Gastroenterology, Shanghai Public Health Clinical Center, Shanghai, China; ${ }^{18}$ Department of Hepatology, Xinjiang Uygur Autonomous Region Traditional Chinese Medicine Hospital, Urumqi, Xinjiang, China; ${ }^{19}$ Department of Infectious Diseases, Shanghai Ruijin Hospital, Jiao Tong University School of Medicine, Shanghai, China;

${ }^{20}$ Department of Infectious Diseases, The First Affiliated Hospital of Xian Jiao Tong University, Xi'an, Shaanxi, China;

${ }^{21}$ Department of Infectious Diseases, Xiangya Hospital Central South University, Changsha, Hunan, China; ${ }^{22}$ Department of Digestive System, Beijing Tiantan Hospital, Capital Medical University, Beijing, China; ${ }^{23}$ Department of Infectious Diseases, Zhongnan Hospital of Wuhan University, Wuhan, Hubei, China; ${ }^{24}$ Department of Infectious Diseases, First Affiliated Hospital of Kunming Medical University, Kunming, Yunnan, China; ${ }^{25}$ Department of Infectious Diseases, The Affiliated Hospital of Xuzhou Medical University, Xuzhou, Henan, China; ${ }^{26}$ Department of Infectious Diseases, Yanbian University Hospital, Yanji, Jilin, China;

${ }^{27}$ Department of Infectious Diseases, The First Affiliated Hospital of Chongqing Medical University, Chongqing, China;

${ }^{28}$ Department of Hepatology, The Fourth People's Hospital of Qinghai Province, Xining, Qinghai, China; ${ }^{29}$ Department of Infectious Diseases, Xinjiang Changji Prefecture People's Hospital, Changji, Xinjiang, China; ${ }^{30}$ Department of Hepatopancreatobiliary Surgery, Affiliated Hospital of Weifang Medical University, Weifang, Shandong, China; ${ }^{31}$ Department of Hepatology, Hunan Provincial People's Hospital, Changsha, Hunan, China; ${ }^{32}$ Department of Gastroenterology, The Second Hospital of Hebei Medical University, Shijiazhuang, Hebei, China; ${ }^{33}$ Department of Hepatology, Xixi Hospital of Hangzhou, Hangzhou, Zhejiang, China;

${ }^{34}$ Department of Infectious Diseases, General Hospital of Ningxia Medical University, Yinchuan, Ningxia, China; ${ }^{35}$ Department of Infectious Diseases, Union Hospital, Tongji Medical College of Huazhong University of Science and Technology, Wuhan, Hubei, China; ${ }^{36}$ Department of Hepatopancreatobiliary and Splenic Medicine, The Affiliated Hospital, Logistics University of People's Armed Police Force, Tianjin, China; ${ }^{37}$ Department of Infectious Diseases, Infectious Disease Hospital of Wuhai, Wuhai, Inner Mongolia, China; ${ }^{38}$ Department of Infectious Diseases, The Affiliated Hospital of Guizhou Medical University, Guiyang, Guizhou, China; ${ }^{39}$ Department of Infectious Diseases, The First Affiliated Hospital of Shanxi Medical University, Taiyuan, Shanxi, China;

${ }^{40}$ Department of Infectious Diseases, The Fifth Hospital of Shijiazhuang, Shijiazhuang, Hebei, China; ${ }^{41}$ Department of Infectious Diseases, Guizhou Provincial People's Hospital, Guiyang, Guizhou, China; ${ }^{42}$ Department of Infectious Diseases, Hainan General Hospital, Haikou, Hainan, China; ${ }^{43}$ Department of Infectious Diseases, The Second Hospital of Shandong University, Jinan,

Shandong, China; ${ }^{44}$ Department of Infectious Diseases, Yanan University Affiliated Hospital, Yan'an, Shaanxi, China;

${ }^{45}$ Department of Infectious Diseases, Peking University First Hospital, Beijing, China; ${ }^{46}$ Peking University Hepatology Institute, Peking University People's Hospital, Beijing, China; ${ }^{47}$ Institute of Hepatology and Department of Infectious Disease, Nanfang Hospital, Southern Medical University, Guangzhou, Guangdong, China; ${ }^{48}$ Artificial Liver Center, Beijing Youan Hospital, Capital 
Shan S. et al: Baseline and treatment for CR-HepB

Medical University, Beijing, China; ${ }^{49}$ Department of Microbiology and Infectious Disease Center, Peking University Health Science Center, Beijing, China; ${ }^{50}$ The China Foundation of Hepatitis Prevention and Control, Beijing China; ${ }^{51}$ Center for Clinical Epidemiology and EBM, Beijing Friendship Hospital, Capital Medical University, National Clinical Research Center for Digestive Diseases, Beijing, China

\section{Abstract}

Background and Aims: Chronic hepatitis B virus (HBV) infection remains a major public health problem globally. Here, we describe the baseline characteristics and treatment profiles of HBV-infected patients recruited to the China Registry of Hepatitis B. Methods: Inclusion criteria were patients with different stages of chronic HBV infection and complete key data. Exclusion criteria were patients with hepatocellular carcinoma. The baseline clinical, laboratory and treatment profiles were analyzed. Results: Finally, 40,431 patients were included. The median age was 43 years, with $65.2 \%$ being men and $51.3 \%$ being positive for hepatitis B e antigen (HBeAg). The most common initial diagnosis was chronic hepatitis $\mathrm{B}(81.0 \%)$, followed by cirrhosis $(9.3 \%)$, inactive carrier of hepatitis B surface antigen ( $\mathrm{HBsAg})(6.7 \%)$, and immune tolerant phase of hepatitis B infection (3.0\%). Among the 21,228 patients who were on treatment, $88.0 \%, 10.0 \%$ and $2.0 \%$ received nucleos(t)ide analogues (NAs), interferon or combination of NAs and interferon, respectively. The proportion of patients who received preferred NAs (entecavir or tenofovir disoproxil fumarate) had increased from $13.5 \%$ in 2003 to $79.7 \%$ in 2016. Conclusions: We concluded that middle-aged men accounted for most of the patients with chronic hepatitis B in this cross-sectional study. About half of the patients were $\mathrm{HBeAg}$-positive. NAs were the most commonly used therapy, and use of the preferred NAs had steadily increased in the past decade.

Citation of this article: Shan S, You H, Niu J, Shan J, Xie W Zhang $Y$, et al. Baseline characteristics and treatment patterns of the patients recruited to the China Registry of Hepatitis B. J Clin Transl Hepatol 2019;7(4):322-328. doi: 10.14218/JCTH.2019.00052.

\section{Introduction}

Universal vaccination against hepatitis B virus (HBV) in infants has achieved great success but chronic HBV infection remains a major public health problem globally. ${ }^{1}$ The 2017 World Health Organization (WHO) Global Hepatitis Report estimates that 257 million persons, or $3.5 \%$ of the population, are chronically infected by $\mathrm{HBV}^{2}$ with the highest hepatitis B surface antigen (HBsAg) prevalence (6.2\%) being in the Western Pacific region. ${ }^{3-5}$ Chronic HBV infection is asso-

\footnotetext{
Keywords: Hepatitis B; Treatment; Registry.
}

Abbreviations: $\mathrm{CHB}$, chronic hepatitis B; CR-HepB, China Registry of Hepatitis B; $\mathrm{HBeAg}$, hepatitis $B$ e-antigen; $\mathrm{HBsAg}$, hepatitis $B$ surface antigen; $\mathrm{HBV}$, hepatitis $B$ virus; HCC, hepatocellular carcinoma; NAs, nucleos(t)ide analogues; WHO, World Health Organization.

Received: 13 October 2019; Revised: 3 December 2019; Accepted: 11 December 2019

* Correspondence to: Jidong Jia, Liver Research Center, Beijing Friendship Hospital, Capital Medical University, 95 Yong-an Road, Xicheng District, Beijing 100050, China. Tel \& Fax: +86-10-63139246, E-mail: jia_jd@ccmu.edu.cn; Yuanyuan Kong, Center for Clinical Epidemiology and EBM, Beijing Friendship Hospital, Capital Medical University, 95 Yong-an Road, Xicheng District, Beijing 100050, China. E-mail: kongyy@ccmu.edu.cn ciated with a considerable burden of liver morbidity and mortality, and can lead to cirrhosis, decompensation and hepatocellular carcinoma (HCC). ${ }^{6}$

In China, with high coverage of HBV vaccination in infants, the estimated prevalence of $\mathrm{HBsAg}$ declined to $6.1 \%$ in the general population. 7,8 However, historical HBV endemicity built a large reservoir of chronically infected persons. It is estimated that there are more than 70 million persons with chronic HBV infection in China. ${ }^{3}$ To facilitate real-world clinical study of chronic HBV infection, we have established a national HBV registry platform, the China Registry of Hepatitis B (known as the CR-HepB), ${ }^{9}$ which was launched in July 2012. Currently, it consists of 47 tertiary hospitals in mainland China (ClinicalTrials.gov registry number: NCT03108794). ${ }^{9}$

In the present cross-sectional study, we described the demographic, baseline characteristics, and treatment profiles of patients recruited in CR-HepB from June 2012 through June 2017.

\section{Methods}

\section{Data sources}

The CR-HepB was launched in June 2012 but retrospectively captured data of patients from 2000. The current study retrieved data from CR-HepB registrants prospectively or retrospectively from June 2012 to June 2017. The key information includes patients' age, gender, diagnosis, laboratory results, liver biopsy results, and antiviral treatment profiles.

\section{Patient population}

Inclusion criteria were patients with different stages of chronic HBV infection and available information on hepatitis $B$ e-antigen (HBeAg) status and HBV DNA and alanine transaminase levels. Exclusion criteria were patients with HCC.

The diagnostic criteria for immune tolerant phase, $\mathrm{HBeAg}$ positive chronic hepatitis $\mathrm{B}(\mathrm{CHB}), \mathrm{HBeAg}$-negative $\mathrm{CHB}$, inactive $\mathrm{HBsAg}$ carriers, cirrhosis, and $\mathrm{HCC}$ were in line with major international and national guidelines ${ }^{10}$ and described in our previous paper. ${ }^{9}$

\section{Statistical analyses}

We use proportions and percentages to describe the demographic and clinical characteristics of the patients. We present the proportions of patients by their age, sex, HBeAg status, diagnosis, liver biopsy results, and type(s) of treatment received. Descriptive statistics are expressed as medians, lower quartiles, and upper quartiles, or as a number and percentage of patients. All statistical analyses were performed using SPSS v19.0.

\section{Results}

After excluding 530 individuals with HCC, 40,431 patients with confirmed diagnoses of immune tolerant phase hepatitis $B$, 
Shan S. et al: Baseline and treatment for CR-HepB

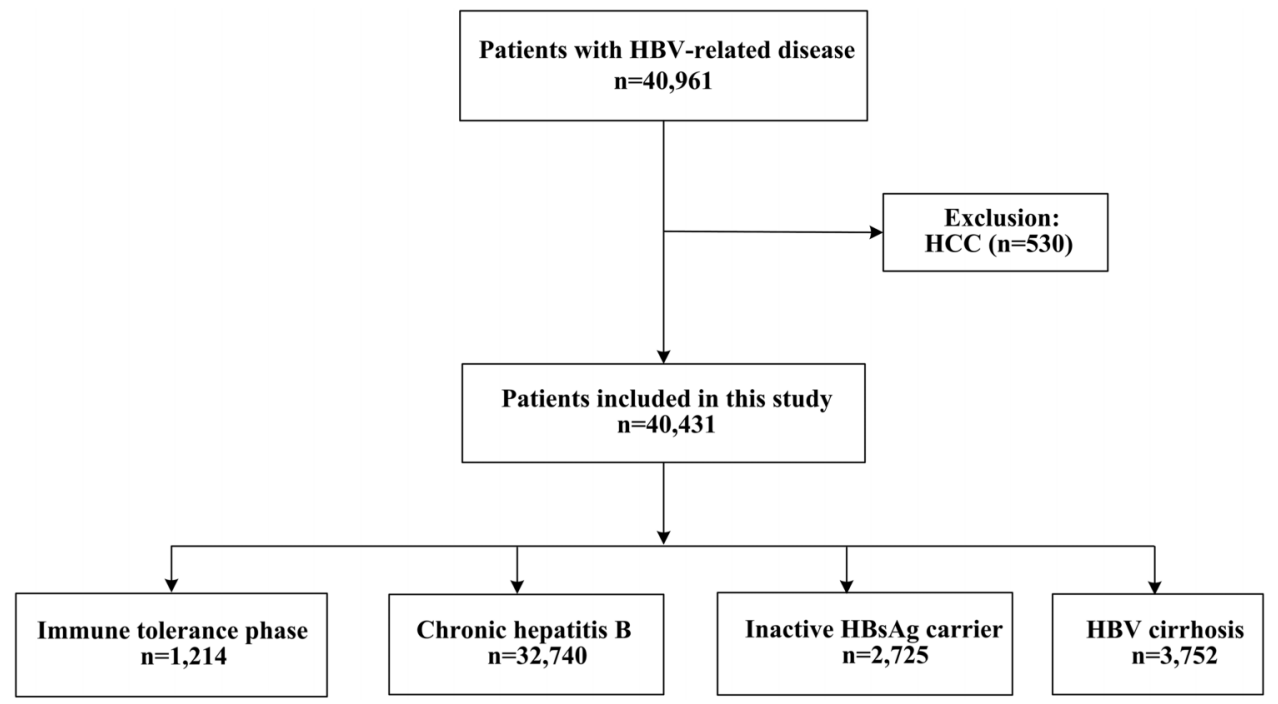

Fig. 1. Flowchart of selection of patients.

$\mathrm{CHB}$, inactive $\mathrm{HBsAg}$ carrier status, and cirrhosis were included in the present study (Fig. 1).

\section{Demographic and clinical characteristics of patients}

The demographic and clinical characteristics of the patients are shown in Table 1 . The median age was 43 years, with a men-to-women ratio of 1.9 . Overall, $51.3 \%$ were $\mathrm{HBeAg}$ positive. Approximately $81.0 \%$ of the patients had initially been diagnosed with $\mathrm{CHB}, 9.3 \%$ with cirrhosis, $6.7 \%$ as inactive HBsAg carriers, and $3.0 \%$ with immune tolerant phase hepatitis $B$.

\section{Age distribution of the 40,431 patients with chronic HBV infection (by sex)}

Among the 40,431 patients included in the present study, the 30-49 years-old age group was the most predominant in both

Table 1. Demographic and baseline data of 40,431 patients with hepatitis $B$ virus-related diseases

\begin{tabular}{|c|c|c|c|c|c|}
\hline & $\begin{array}{l}\text { Overall, } \\
n=40,431\end{array}$ & $\begin{array}{l}\text { Immune } \\
\text { tolerance } \\
\text { phase, } n=1,214\end{array}$ & $\begin{array}{l}\text { Inactive } \\
\text { HBsAg carrier, } \\
n=2,725\end{array}$ & $\begin{array}{l}\text { Chronic hepatitis } \\
\mathrm{B}, n=32,740\end{array}$ & $\begin{array}{l}\text { Cirrhosis, } \\
n=3,752\end{array}$ \\
\hline Age in years & $43(33,53)$ & $33(28,41)$ & $39(31,49)$ & $43(33,52)$ & $55(48,63)$ \\
\hline \multicolumn{6}{|l|}{ Sex } \\
\hline Men, $n(\%)$ & $26,347(65.2)$ & $610(50.2)$ & $1522(55.9)$ & $21,472(65.6)$ & $2743(73.1)$ \\
\hline Women, $n(\%)$ & $14084(34.8)$ & $604(49.8)$ & $1203(44.1)$ & $11,268(34.4)$ & 1,009 (26.9) \\
\hline $\begin{array}{l}\text { HBeAg-positive, } \\
n(\%)\end{array}$ & $20740(51.3)$ & $1214(100.0)$ & $0(0)$ & $17936(54.8)$ & $1590(42.4)$ \\
\hline $\begin{array}{l}\text { HBV DNA } \\
\left(\log _{10} \mathrm{IU} / \mathrm{mL}\right)\end{array}$ & $3.9(2.3,6.6)$ & $7.6(6.3,8.1)$ & $0(0,2.6)$ & $4.2(2.7,6.9)$ & $3.9(2.0,5.6)$ \\
\hline ALT (IU/mL) & $41.7(24.6,87.0)$ & $27.0(21.0,34.8)$ & $24.0(18.0,33.0)$ & $46.0(26.0,99.0)$ & $42.0(27.0,76.0)$ \\
\hline AST (IU/mL) & $34.0(23.0,63.0)$ & $22.0(17.0,26.0)$ & $23.0(19.0,28.0)$ & $35.6(24.067 .2)$ & $47.0(29.5 .0,83.6)$ \\
\hline ALP (U/L) & $78.0(62.0,101.0)$ & $69.0(56.0,85.0)$ & $70.0(58.0,86.0)$ & $77.7(62.0,99.0)$ & $97.8(73.0,133.0)$ \\
\hline GGT (U/L) & $27.0(16.0,55.0)$ & $15.0(12.0,21.0)$ & $17.0(12.0,25.0)$ & $28.0(17.0,55.2)$ & $50.9(27.9,99.0)$ \\
\hline $\begin{array}{l}\text { Bilirubin } \\
(\mu \mathrm{mol} / \mathrm{L})\end{array}$ & $14.8(10.9,21.5)$ & $12.1(9.3,15.8)$ & $12.8(9.7,17.2)$ & $14.4(10.8,20.3)$ & $24.5(15.9,41.3)$ \\
\hline ALB $(\mathrm{g} / \mathrm{L})$ & $44.0(39.7,46.7)$ & $45.2(43.2,47.3)$ & $45.4(43.4,47.4)$ & $44.2(40.4,46.9)$ & $34.8(29.4,41.2)$ \\
\hline $\begin{array}{l}\text { PLT count } \\
\left(\times 10^{9} / \mathrm{L}\right)\end{array}$ & $\begin{array}{l}165.0(115.0 \\
208.3)\end{array}$ & $\begin{array}{l}200.0(173.0 \\
236.0)\end{array}$ & $\begin{array}{l}188.0(154.0, \\
225.0)\end{array}$ & $\begin{array}{l}171.0(129.0 \\
211.0)\end{array}$ & $81.0(53.0,123.0)$ \\
\hline
\end{tabular}

Data are expressed as median (range) or $n(\%)$.

Abbreviations: ALB, albumin; ALT, alanine aminotransferase; AST, aspartate aminotransferase; HBeAg, hepatitis B e antigen; HBsAg, hepatitis B surface antigen; HBV, hepatitis B virus; PLT, platelet. 
Shan S. et al: Baseline and treatment for CR-HepB

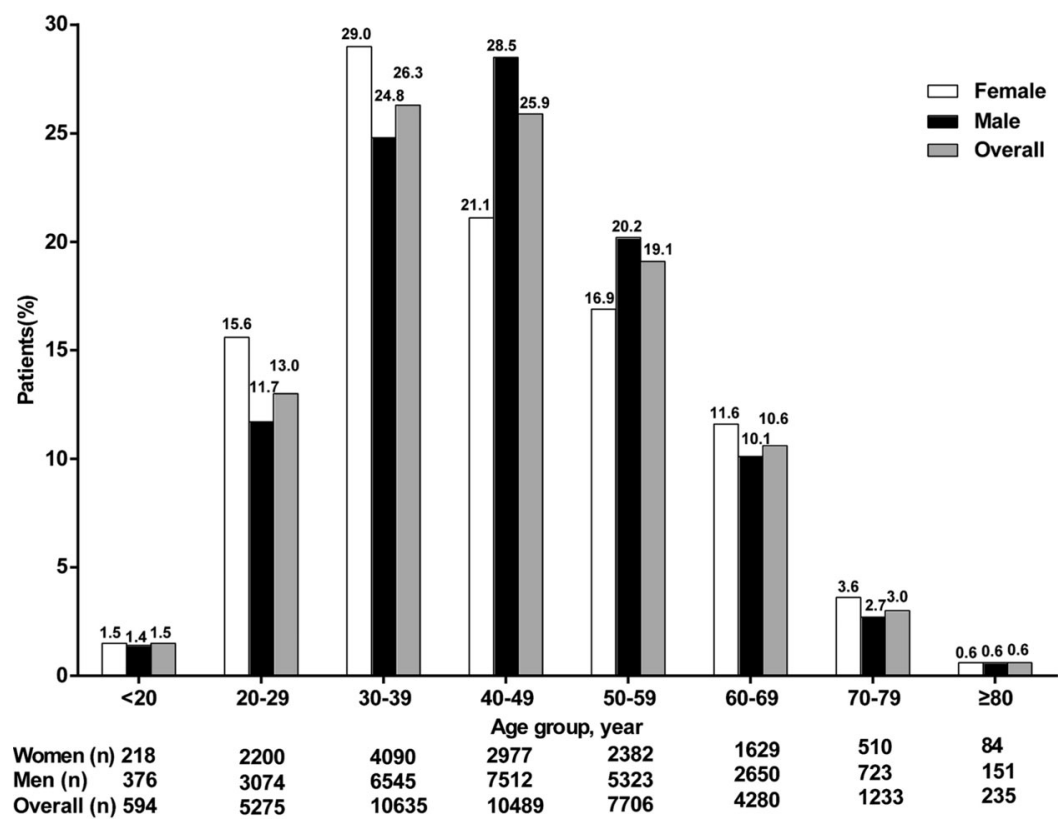

Fig. 2. Age distribution of chronic hepatitis $B$ virus infection by sex in 40,431 patients.

men and women, followed by the 50-59 years-old age group. Patients aged between $30-59$ years-old accounted for $71.8 \%$ of all patients (Fig. 2).

\section{Disease distribution in different age groups among the 40,431 patients}

The proportion of patients diagnosed with cirrhosis was increased with increasing ages, whereas the proportion of patients diagnosed with immune tolerance phase was decreased with increasing age (Fig. 3).

\section{Liver histology of 485 patients}

Necroinflammation activity and fibrosis stage were assessed according to the Scheuer grading and staging system. ${ }^{11}$
Among the 485 patients who underwent liver biopsy, the proportion of patients with liver inflammation grade $\geq 2$ or the stage of liver fibrosis $\geq 2$ increased with age (Fig. 4).

\section{Treatment profiles of 21,228 patients and the changing prescription of different nucleos $(t)$ ide analogues}

Nucleos(t)ide analogues (NAs) were the most common therapy among the 21,228 patients with prescription information. A much smaller proportion of patients were treated with interferon $(10.0 \%)$ or a combination of interferon and NAs $(2.0 \%)$.

Lamivudine (15.3\%) and adefovir dipivoxil (18.4\%) were widely used before 2011, whereas the use of entecavir (51.4\%) and tenofovir disoproxil fumarate (2.1\%) dramatically increased after 2011 (Fig. 5).

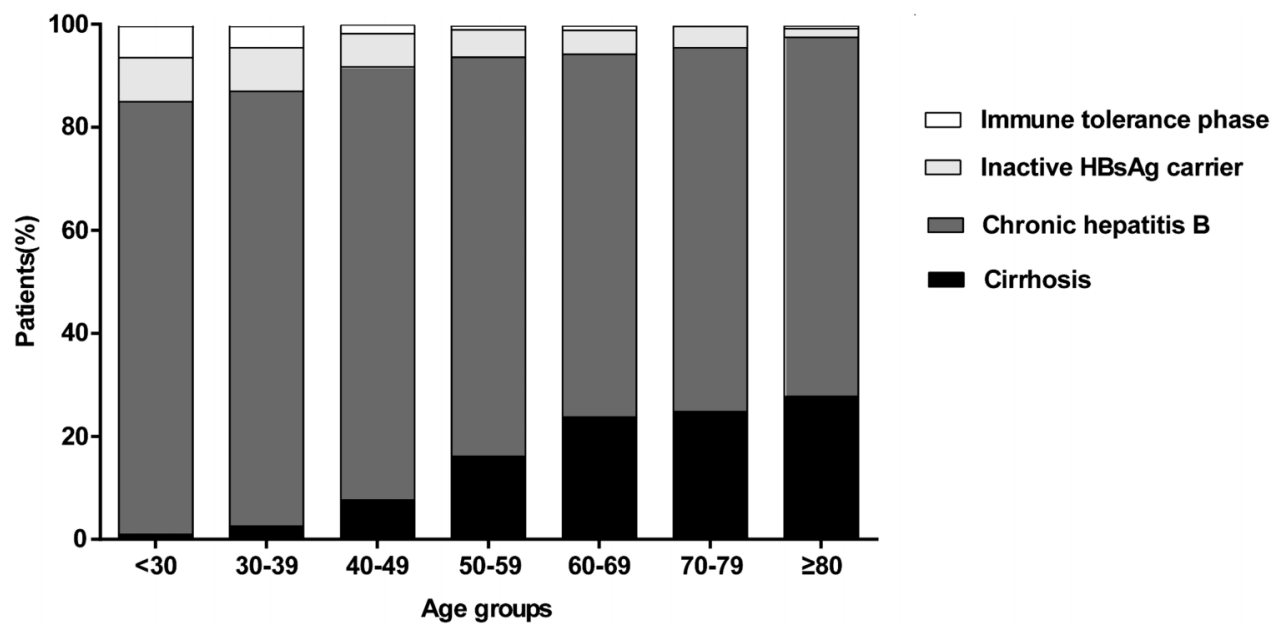

Fig. 3. Disease distribution in different age groups among the 40,431 patients. 
Shan S. et al: Baseline and treatment for CR-HepB
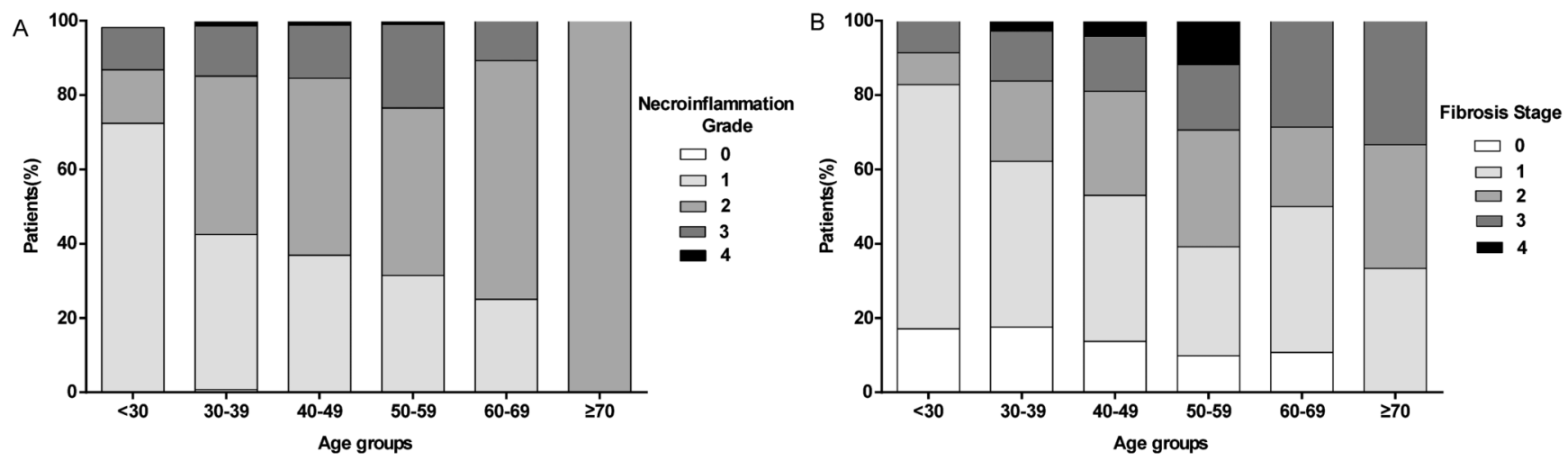

Fig. 4. Distribution of (A) the necroinflammation grade and (B) fibrosis stage in 485 patients.

\section{Discussion}

In the present study, with large number of patients, we found that middle-aged men represented the major proportion of this cohort. About half of the patients were HBeAg-positive. The most common initial diagnosis was $\mathrm{CHB}$, followed by cirrhosis, inactive HBsAg carrier, and immune tolerant phase of hepatitis $B$ infection. The proportion of patients diagnosed with cirrhosis was increasing with increasing age. Among the patients with prescription information, nearly $90 \%$ received NAs and the use of preferred NAs have increased dramatically in the past decade.

Our study showed that men accounted for a significantly higher proportion (65.2\%) than women (34.8\%), and about half of patients were HBeAg-positive. This is similar to the result of a recent multicenter, real-world study conducted in tier-2 city hospitals in China, which showed that $74 \%$ of 3,408 patients with $\mathrm{CHB}$ were men, with an overall mean age of 40 years, and that $60 \%$ of patients were HBeAg-positive. ${ }^{12}$ Not surprisingly, patients with $\mathrm{HBeAg-negative} \mathrm{infection} \mathrm{were}$ older than those with $\mathrm{HBeAg}$-positive infection, also similar to that reported from the USA. ${ }^{13}$
In our study, the middle-aged group was the most predominant in both men and women. This is in line with the recent reports that the prevalence of $\mathrm{HBsAg}$ in childbearingaged men and women still being around $6 \%$ in rural and endemic areas in China. ${ }^{14}$ Therefore, prevention of motherto-child transmission is still of paramount importance. ${ }^{3,15,16}$

Not surprisingly, the proportion of patients diagnosed with cirrhosis was increased with increasing age. Similarly, among the 485 patients who underwent liver biopsy, the majority of these patients had mild to moderate necroinflammation and fibrosis. This may be due to the fact that patients with more disease activity and advanced fibrosis could be identified easily by noninvasive modalities, making them under-represented among patients who received liver biopsy.

In our study, more than half of the patients were prescribed treatment, and nearly $90 \%$ of them received NAs due to their favorable efficacy and safety as well as ease of administration. All major international guidelines recommend highly potent entecavir and tenofovir disoproxil fumarate as preferred therapy, ${ }^{10,17-19}$ since accumulating evidence indicates that long-term therapy with entecavir or tenofovir disoproxil fumarate can prevent or reverse liver fibrosis and

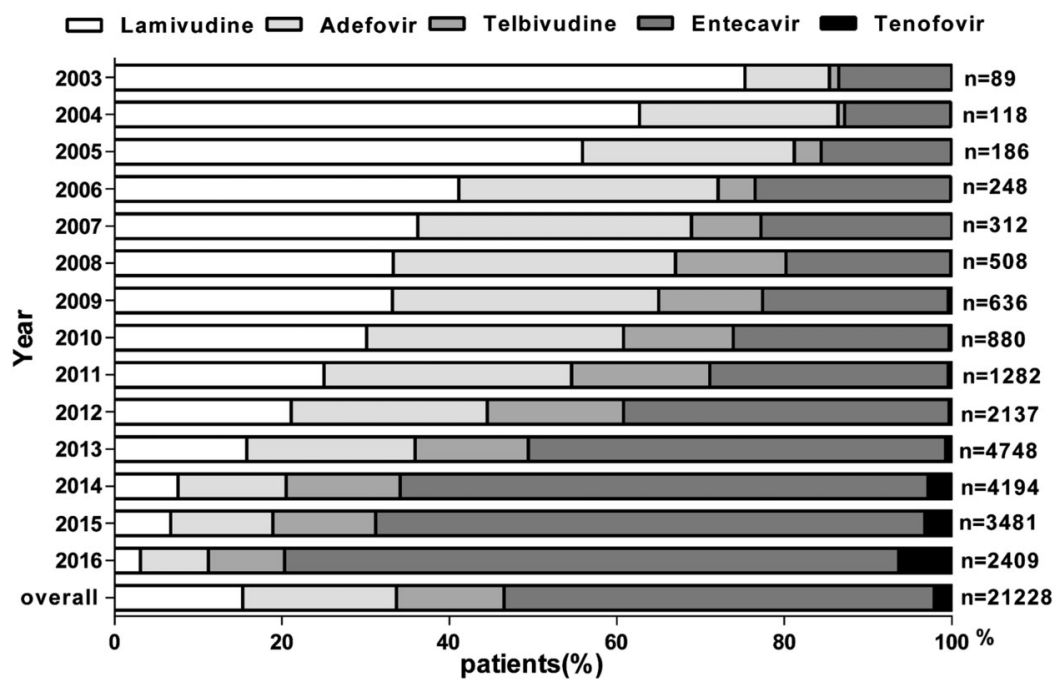

Fig. 5. Changes in proportion of each nucleos(t)ide analogue use, 2003-2016. 
reduce risk of HCC. ${ }^{20-22}$ However, in real-world practice, lots of patients had been treated with lamivudine, adefovir dipivoxil and telbivudine, which are not preferred therapy, due to their low antiviral potency and low genetic barrier. This discrepancy between guideline recommendations and real-world clinical practice may be influenced by many factors, including doctors' knowledge, reimbursement policy, and patients' economic status and compliance. ${ }^{22-24}$

Fortunately, this study showed the prescription of different NAs has favorably changed in the past years, with entecavir prescription increased from less than one-third to more than half. This trend may reflect the following facts: 1 ) evidence from clinical trial and real-world studies convincingly demonstrates the efficacy and safety of antiviral therapy, ${ }^{12,25,26} 2$ ) update of evidence-based national guidelines recommends entecavir and tenofovir disoproxil fumarate as first-line therapy, ${ }^{10} 3$ ) evolving national and local reimbursement policy offers more potent antiviral therapy for people who are covered by basic social medical insurance. All these improved the standard of care in clinical practice for CHB treatment. Tenofovir disoproxil fumarate was used only in less than $10 \%$, simply because it had not been proved for HBV until mid-2014 in mainland China.

We hope this large nationwide database could provide a point of view of clinical profiles of chronic HBV infection and the treatment landscape in mainland China. However, several limitations in our study need to be mentioned. First, since CRHepB is a hospital-based registry system, the proportion of inactive HBsAg carriers may be underestimated, as these patients are usually asymptomatic and may not seek medical service. Second, the cross-sectional design made it difficult to identify factors associated with disease progression or regression. However, CR-HepB registrants are advised to received follow-up visits every 3 to 6 months, so we could expect this limitation may be solved in the future. A final limitation is potential selection bias, as the majority of patients in the CRHepB are from tertiary hospitals, therefore not necessarily reflecting the clinical practice in secondary or primary medical care settings where the resources and expertise are far less privileged.

\section{Conclusions}

In conclusion, this hospital-based cross-sectional study provides a snapshot of demographic and baseline profiles of Chinese patients with different stage of chronic HBV infection, as well as the landscape of clinical management.

\section{Ethics Approval}

The registry protocol was reviewed and approved by the ethics committee of Beijing Friendship Hospital, Capital Medical University (Approval Number: BJFH-EC/2014-044). Each participating institution also obtained approval from its institutional ethics committee.

\section{Acknowledgments}

We thank the China Foundation for Hepatitis Prevention and Control, Chinese Society of Hepatology, Chia Tai-Tianqing Pharmaceutical Group Co., Ltd and Shanghai Ashermed Healthcare Communications, Ltd, for their administrative coordination, technical assistance, and unrestricted grant to the CR-HepB and this paper.

\section{Conflict of interest}

The authors have no conflict of interests related to this publication.

\section{Author contributions}

Designed the study (JJ, HY, HZ, LW, JH, ZD), drafted the manuscript (SS), data management (YK). Served as the project leader and extensively and critically revised this manuscript (JJ). The other authors are the team members. All authors have read and approved the final version of the manuscript.

\section{References}

[1] Naghavi $M$, Wang $H$, Lozano $R$, Davis $A$, Liang $X$, Zhou $M$, et al. Global, regional, and national age-sex specific all-cause and cause-specific mortality for 240 causes of death, 1990-2013: a systematic analysis for the Global Burden of Disease Study 2013. Lancet 2015;385:117-171. doi: 10. 1016/S0140-6736(14)61682-2.

[2] World Health Organization. Global hepatitis report, 2017. Available from: https://apps.who.int/iris/bitstream/handle/10665/255016/9789241565455-eng.pdf;jsessionid=7DA50E712691FED09098855C3796A3A7? sequence $=1$.

[3] Schweitzer A, Horn J, Mikolajczyk RT, Krause G, Ott J]. Estimations of worldwide prevalence of chronic hepatitis $B$ virus infection: a systematic review of data published between 1965 and 2013. Lancet 2015;386:1546-1555. doi: $10.1016 / \mathrm{S} 0140-6736(15) 61412-X$

[4] Ott J], Stevens GA, Groeger ], Wiersma ST. Global epidemiology of hepatitis $B$ virus infection: new estimates of age-specific $\mathrm{HBsAg}$ seroprevalence and endemicity. Vaccine 2012;30:2212-2219. doi: 10.1016/j.vaccine.2011.12. 116.

[5] Tian Q, Jia J. Hepatitis B virus genotypes: epidemiological and clinical relevance in Asia. Hepatol Int 2016;10:854-860. doi: 10.1007/s12072-0169745-2.

[6] Stanaway JD, Flaxman AD, Naghavi M, Fitzmaurice C, Vos T, Abubakar I, et al. The global burden of viral hepatitis from 1990 to 2013: findings from the Global Burden of Disease Study 2013. Lancet 2016;388:1081-1088. doi: 10.1016/S0140-6736(16)30579-7.

[7] Liang X, Bi S, Yang W, Wang L, Cui G, Cui F, et al. Reprint of: Epidemiological serosurvey of Hepatitis $B$ in China-declining HBV prevalence due to Hepatitis B vaccination. Vaccine 2013;31 Suppl 9:J21-J28. doi: 10.1016/j.vaccine. 2013.08.012.

[8] Cui F, Shen L, Li L, Wang H, Wang F, Bi S, et al. Prevention of chronic hepatitis $B$ after 3 decades of escalating vaccination policy, China. Emerg Infect Dis 2017;23:765-772. doi: 10.3201/eid2305.161477.

[9] Shan S, Wei W, Kong Y, Niu J, Shang J, Xie W, et al. China Registry of Hepatitis B (CR-HepB): Protocol and implementation of a nationwide hospital-based registry of hepatitis B. Scand J Public Health 2018:1403494818772188. doi: $10.1177 / 1403494818772188$.

[10] Hou J, Wang G, Wang $F$, Cheng J, Ren $\mathrm{H}$, Zhuang $\mathrm{H}$, et al. Guideline of prevention and treatment for chronic hepatitis B (2015 update). J Clin Transl Hepatol 2017;5:297-318. doi: 10.14218/JCTH.2016.00019.

[11] Scheuer PJ. Classification of chronic viral hepatitis: a need for reassessment. J Hepatol 1991;13:372-374. doi: 10.1016/0168-8278(91)90084-o.

[12] Jia J, Tang H, Ning Q, Jiang J, Dou X, Zhang $M$, et al. Real-world evidence for nucleoside/nucleotide analogues in a 5-year multicentre study of antiviralnaive chronic hepatitis B patients in China: 52-week results. Antivir Ther 2018;23:201-209. doi: 10.3851/IMP3205.

[13] Spradling PR, Xing J, Rupp LB, Moorman AC, Gordon SC, Teshale ET, et al. Distribution of disease phase, treatment prescription and severe liver disease among 1598 patients with chronic hepatitis B in the Chronic Hepatitis Cohort Study, 2006-2013. Aliment Pharmacol Ther 2016;44:1080-1089. doi: 10.1111 /apt.13802.

[14] He T, Jia J. Chronic HBV: which pregnant women should be treated? Liver Int 2016;36 Suppl 1:105-108. doi: 10.1111/liv.13010.

[15] Cui F, Liang X, Gong X, Chen Y, Wang F, Zheng H, et al. Preventing hepatitis B though universal vaccination: reduction of inequalities through the GAVI China project. Vaccine 2013;31 Suppl 9:J29-J35. doi: 10.1016/j.vaccine. 2012.07.048. 
[16] Liu J, Zhang S, Wang $\mathrm{O}$, Shen $\mathrm{H}$, Zhang $M$, Zhang $Y$, et al. Seroepidemiology of hepatitis B virus infection in 2 million men aged 21-49 years in rural China: a population-based, cross-sectional study. Lancet Infect Dis 2016; 16:80-86. doi: 10.1016/S1473-3099(15)00218-2.

[17] Terrault NA, Lok ASF, McMahon BJ, Chang KM, Hwang JP, Jonas MM, et al. Update on prevention, diagnosis, and treatment of chronic hepatitis $B$ : AASLD 2018 hepatitis B guidance. Hepatology 2018;67:1560-1599. doi: 10.1002/hep. 29800.

[18] Lampertico P, Agarwal K, Berg T, Buti M, Janssen HLA, Papatheodoridis G, et al. EASL 2017 Clinical Practice Guidelines on the management of hepatitis B virus infection. J Hepatol 2017;67:370-398. doi: 10.1016/j.jhep.2017.03. 021.

[19] Sarin SK, Kumar M, Lau GK, Abbas Z, Chan HL, Chen CJ, et al. Asian-Pacific clinical practice guidelines on the management of hepatitis B: a 2015 update. Hepatol Int 2016;10:1-98. doi: 10.1007/s12072-015-9675-4.

[20] Varbobitis I, Papatheodoridis GV. The assessment of hepatocellular carcinoma risk in patients with chronic hepatitis B under antiviral therapy. Clin Mol Hepatol 2016;22:319-326. doi: 10.3350/cmh.2016.0045.
[21] Papatheodoridis GV, Chan $\mathrm{HL}$, Hansen BE, Janssen $\mathrm{HL}$, Lampertico P. Risk of hepatocellular carcinoma in chronic hepatitis B: assessment and modification with current antiviral therapy. J Hepatol 2015;62:956-967. doi: 10. 1016/j.jhep.2015.01.002.

[22] Sun Y, Zhou J, Wang L, Wu X, Chen Y, Piao H, et al. New classification of liver biopsy assessment for fibrosis in chronic hepatitis $B$ patients before and after treatment. Hepatology 2017;65:1438-1450. doi: 10.1002/hep.29009.

[23] Lim SG, Amarapurkar DN, Chan HL, Crawford DH, Gane EJ, Han KH, et al. Reimbursement policies in the Asia-Pacific for chronic hepatitis B. Hepatol Int 2015;9:43-51. doi: 10.1007/s12072-014-9593-x.

[24] Shan S, Cui F, Jia J. How to control highly endemic hepatitis B in Asia. Liver Int 2018;38 Suppl 1:122-125. doi: 10.1111/liv.13625.

[25] Wei L, Jia JD, Weng XH, Dou XG, Jiang JJ, Tang $H$, et al. Treating chronic hepatitis B virus: Chinese physicians' awareness of the 2010 guidelines. World J Hepatol 2016;8:762-769. doi: 10.4254/wjh.v8.i18.762.

[26] Zeng N, Zou C, He Z, Ma H, Ou X, You H, et al. Systematic review on the reporting quality of randomized controlled trials in patients with hepatitis $\mathrm{B}$ or C in China. Int J Infect Dis 2018;67:58-64. doi: 10.1016/j.ijid.2017.11.011. 